\title{
Management of nausea and vomiting in pregnancy
}

This clinical review by Sheba Jarvis and Catherine Nelson-Piercy wrongly refers to "dopamine agonists" in two places (BMJ 2011;342:d3606, doi:10.1136/bmj.d3606). In the summary points box and the "Antiemetics" section, the authors should have said "dopamine antagonists."

Cite this as: $B M J$ 2011;342:d4018 